\title{
The use of an ultrasonic monitor for recording locomotor activity
}

\author{
W. HINANO AKAKA and BECKY A. HOUCK \\ Békésy Laboratory of Neurobiology, University of Hawaii, Honolulu, Hawaii 96822
}

\begin{abstract}
An ultrasonic activity monitor has been designed that can be used to detect motion in air or within an aquarium. Stability and linearity of response of the monitor have been measured. The system has been used to examine activity rhythms of the marine invertebrate, Octoput ornatus Gould.
\end{abstract}

An ultrasonic motion detector can monitor wideranging locomotor activity of an animal without imposing restrictions on movement or disturbing normal behavior. The fact that such a system has not often been used in studies of locomotor activity can be attributed partially to the cost of portions of the system, such as the ultrasonic transducers, and to the lack of information on the problems and calibration of an ultrasonic system.

The ultrasonic motion detector described here is a modification of the system described by Meffert (1968). The primary contribution of the present system involves adaptation of inexpensive, commercially available transducers for use in either an air-filled enclosure or with a fresh or salt water aquarium or tank. The modified transducers can be used external to the recording portion of the tank or enclosure, extending the operating life of the system indefinitely. The locomotor activity detector was calibrated according to uniformity of response within the test enclosure, linearity of response with varying amplitudes of activity, and stability over time.

\section{ULTRASONIC MONITORING SYSTEM}

An ultrasonic monitoring device consists of an audio oscillator that produces an ultrasonic signal, a transmitting transducer, a receiving transducer, a detector receiving input about both the transmitted and received signals, a counter, a clock, and a printer or data storage device.

In theory, the monitor compares the transmitted and received ultrasonic signals. An ultrasonic standing wave is produced in a closed chamber, and when the sound field is stable, the amplitude of the received signal is constant. If an animal is moving in the chamber, the amplitude of the received signal varies in proportion to the size and velocity of the movement.

A block diagram of the monitoring system is provided in Figure 1, and the circuitry is diagrammed in

This work was supported in part by an NIH predoctoral fellowship to B. A. Houck, whose present address is the Department of Physical and Life Sciences, University of Portland, Portland. Oregon 97203.
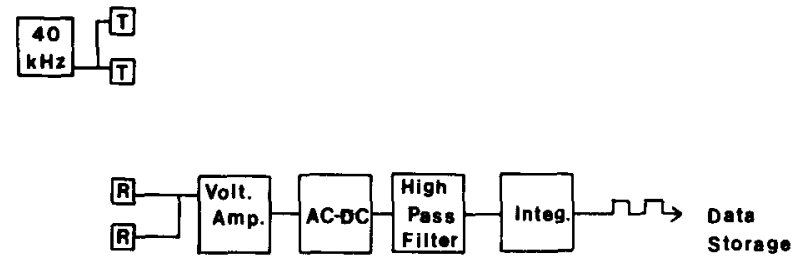

Figure 1. Block diagram of the ultrasonic activity monitor.

Figure 2. The ultrasonic signal is produced by an audio oscillator and transmitted to a tank via ceramic transducers. Two transmitting and two receiving transducers are placed on the Plexiglas front panel of marine plywood tanks. Since the Plexiglas is acoustically transparent, the transducers do not have to contact the salt water of the tank enclosure. The signal is detected by the receiving transducers. The output voltage of the receiving transducers is amplified to a $1 \cdot V$ peak-to-peak signal, which is fed to an ac/dc converter acting as a demodulator. The dc component is high-pass filtered with a cutoff frequency of $.1 \mathrm{~Hz}$ to eliminate lowfrequency drift. The signal was integrated by a voltagecontrolled oscillator, and the summed responses over 2-min intervals, indicating activity over that period, are read directly by a Digital Equipment Corporation PDP-11/10 computer. The activity values are stored on digital cassettes, along with information on the time and status of the animal. This information can be read off the tape by the computer and analyzed at a later time.

The transmitting and receiving transducers are Calectro ultrasonic transducers, available at Lafayette Radio Stores for use in room burglar alarm systems at a 1979 cost of less than $\$ 10$ each. The transducers and the electrical connections are protected from the corrosive action of salt water by placement in a plastic housing. The ceramics are removed from the metal casing and sealed in a PVC pipe of $2.54 \mathrm{~cm}$ inner diameter. The pipe is modified as shown in Figure 3. A lip is produced on the inner surface, against which the ceramic is sealed with silastic. The connector on the transducing unit is replaced by coaxial cable, and the entire space behind the ceramic is filled with silastic. After curing, the modified transducing unit is ready to 


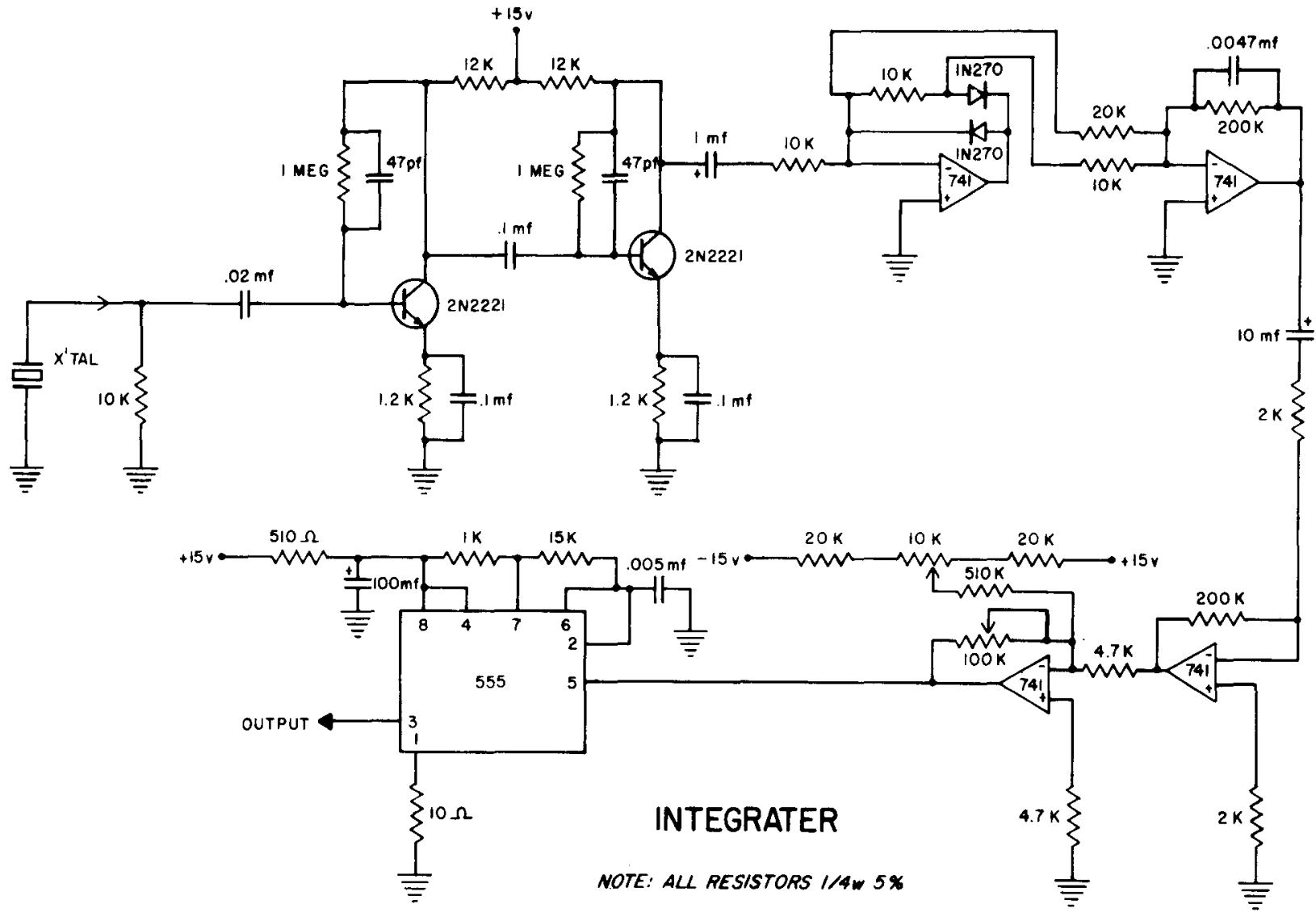

Figure 2. Circuit diagram of the monitor.

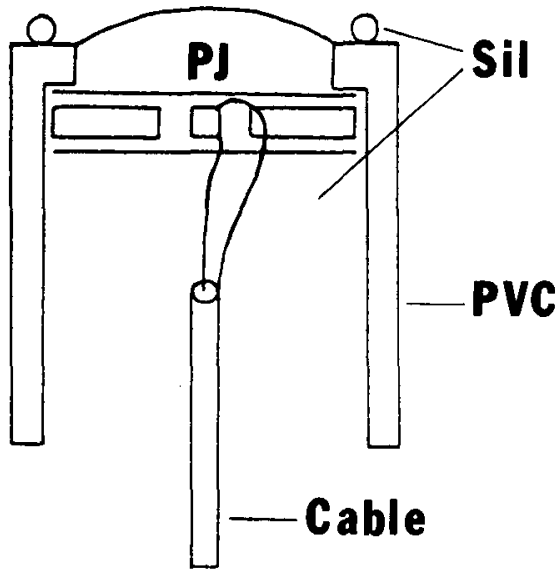

Figure 3. Modification of the ultrasonic transducer for use with a marine aquarium. $\mathrm{PJ}=$ petroleum jelly; $\mathrm{Sil}=$ silastic; PVC = PVC pipe.

be mounted. The space between the ceramic and the tank wall is filled with petroleum jelly; care is taken not to allow any on the rim of the PVC pipe. Silastic is placed on the rim of the pipe, and the unit is pressed against the Plexiglas wall of the tank. The silastic provides the adhesion, and the petroleum jelly acoustically couples the ceramic to the tank.

\section{CALIBRATION}

Uniformity of the Sound Field in the Tanks

The tanks were tested with a 2-rpm motor attached to a rod on which was placed a Plexiglas paddle. This mimicked a "standard" activity level that could be moved to various positions in the tank to test uniformity. Values obtained from 12 positions in each of two tanks produced a coefficient of variation of $25.6 \%$ in one tank and $27.8 \%$ in the other. Hence the sound field is not uniform throughout the tank, and such variation constitutes a significant concern in the use of an ultrasonic monitor.

\section{Linearity of Response Characteristics}

A variable-speed motor was used with the paddle set-up described above to examine the linearity of response of the monitor. Revolutions per minute were increased, and standard activity was measured at each speed. A least-squares plot is drawn through the mean data points obtained (Figure 4). The response is linear over the range tested.

\section{Stability Over Time}

Standard activity was measured in the tanks over a 


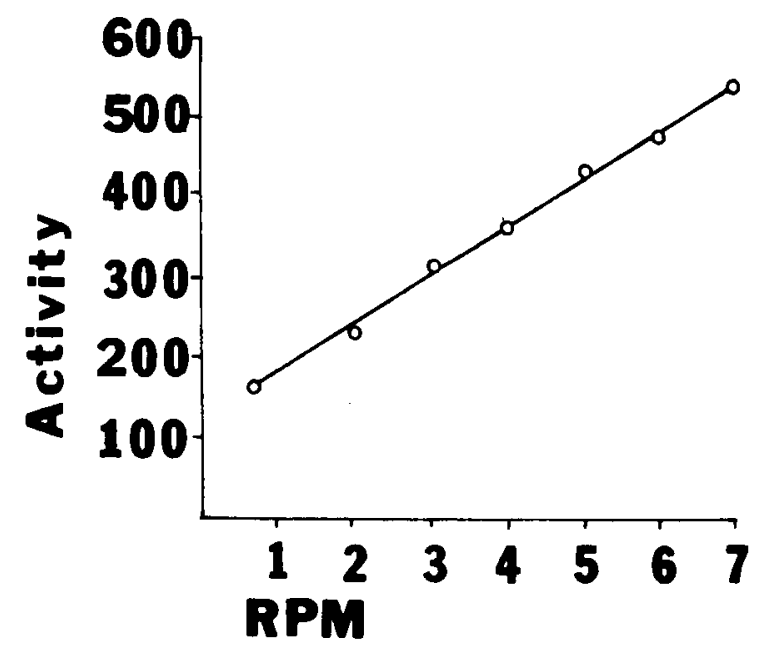

Figure 4. Standard activity with increasing rpms.

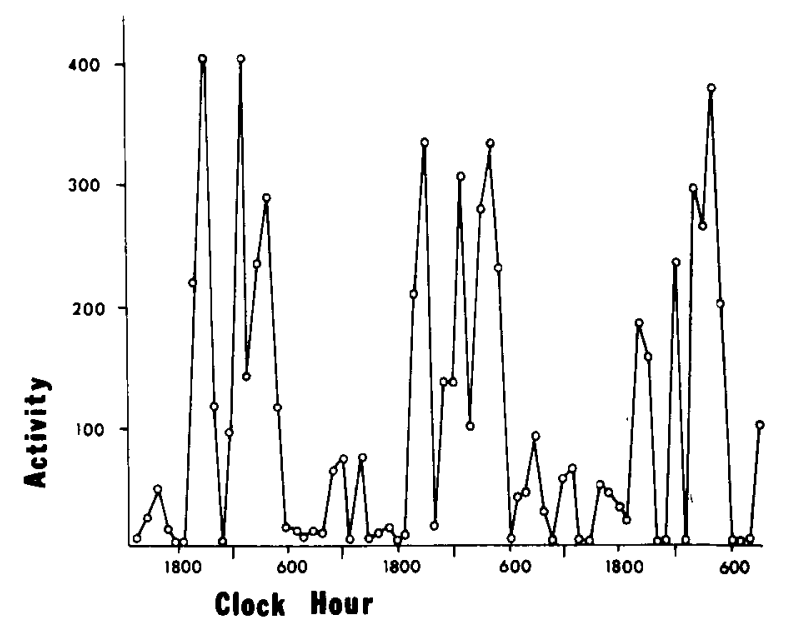

Figure 5. Natural activity of Octopus ornatus in darkness. $N=68$ hourly values.

1-year period. When the oscillators were tuned to produce maximum $(1 . \mathrm{V})$ response, the system was completely stable and the same activity values were obtained. However, the transducer ceramics altered their optimum oscillating frequency over time, indicating that the tuning frequency of such a system should be monitored periodically.

\section{APPLICATION}

The ultrasonic activity monitor described here was used in the study of the circadian activity rhythm of a marine invertebrate Octopus oranatus Gould. An example of a "run" in which the animal was placed in darkness and left undisturbed for a period of several days is shown in Figure 5. Hours corresponding to dawn and dusk in the field are marked.

One problem in using the ultrasonic monitor is the interaction between the size of the animal tested and the amplitude of the recorded activity. This does not affect examination of individual records for one animal, but comparison of the activity of animals of differing size becomes difficult. Since the mean activity of any run was directly proportional to the biological variable (the weight of the animal), activity values were standardized by division of raw hourly values by the mean of the run. The quotient was expressed as a percentage. In this way, an hourly value equal to the hourly mean of the monitoring period gave a percentage of $100 \%$, regardless of the magnitude of the raw values. With this measure, the animal serves as a standard against itself, and animals of varying sizes can be compared with each other. In addition, the procedure minimizes any changes in the stability of the system over long periods of time.

The monitored activity of $O$. ornatus shows a distinct circadian rhythmicity. Maximum activity occurs during the subjective nocturnal hours (hours of expected darkness), and limited activity occurs during subjective daylight hours. Although no photic cues were provided for the animal during the monitoring period, the octopus demonstrates locomotor activity that correlates with environmental photic variations in the field. The ultra sonic activity monitor is effective in accurately recording the locomotor activity of $O$. ornatus without imposing restrictions on movement of the experimental animal within a laboratory aquarium.

\section{REFERENCE}

Meffert, P. Ultrasonic recorder for locomotor activity studies. Transactions American Fisheries Society, 1968, 97, 12-17.

(Received for publication May 27, 1979; revision accepted July 25,1980 .) 